\title{
On Solutions and Heteroclinic Orbits of Some Lotka-Volterra Systems
}

\author{
Supriya Mandal ${ }^{1}$, M. M. Panja ${ }^{2}$, Santanu Ray ${ }^{3}$ \\ ${ }^{1,2}$ Department of Mathematics, Visva-Bharati (A Central University), Santiniketan-731235, West Bengal, India \\ ${ }^{3}$ Department of Zoology, Visva-Bharati (A Central University), Santiniketan-731235, West Bengal, India \\ ${ }^{1}$ Email addresses-supriyamandal81@gmail.com \\ 2Email addresses-madanpanja2005@yahoo.co.in \\ ${ }^{3}$ Email addresses-sray@visva-bharati.ac.in \\ ${ }^{2}$ Corresponding Author
}

\begin{abstract}
In this work, a principle for getting heteroclinic orbit of a dynamical system has been proposed when the solution is known in a compact form. The proposed principle has been tested through its application to a three species Lotka-Volterra system, which may appear as a mathematical model of human pathogen system. The domain in parameter $(a, \lambda)$ space involve in the model, and the region of initial condition $\mathbb{H}=$ $\left\{(x(0), y(0), z(0)) \in \mathbb{R}^{3}\right\} \subset \mathbb{R}^{3}$ for the existence of heteroclinic orbit have been derived.
\end{abstract}

Indexing terms/Keywords: LV system, Exact solution, Heteroclinic orbit, Invariant.

\section{Subject Classification:}

\section{Type (Method/Approach):}

Supporting Agencies:

Language: English

Date of Publication: 2018-07-30

DOI: 10.24297/jam.v14i2.7499

ISSN: 2347-1921

Volume: 14 Issue: 02

Journal: Journal of Advances in Mathematics

Publisher: CIRWORLD

Website: https://cirworld.com

This work is licensed under a Creative Commons Attribution 4.0 International License. 


\section{Introduction}

Lotka-Volterra (LV) system is one of the widely used models in the field of nonlinear dynamics. This system with three or higher species can describe many scientific phenomena in the arena of science and engineering even in social sciences[1-2]. Like most of the other nonlinear systems, the exact solution of differential equations involved in the LV system is very hard to get when the number of species is large.

Heteroclinic orbits are of great importance in the study of dynamical systems [3 -4]. Finding heteroclinic or homoclinic orbits for a given dynamical system is not an easy task. But their presence provides much information about the behaviour of the system. Li et.al.[5] presented the condition of existence/non-existence of homoclinic or heteroclinic orbit of Chen system. Tigan[6] presented a method to detect homoclinic or heteroclinic orbit of a three-dimensional dynamical system by tracing the separatrices until they hit a given surface and then looking for the condition such that the separatrices meet one another on this surface. The varied heteroclinic orbit was used to make the late-time behaviour of the model insensitive to the initial condition and thus alleviating the fine-tuning problem in the cosmological dynamical system of a barotropic fluid and quintessence with a double exponential problem[7]. Beiye presented sufficient condition for the existence of heteroclinic cycles of $n$-species LV system and criterion for determining the stability of heteroclinic cycles[8]. A series method for continuous-time autonomous dynamical systems was proposed to find exact heteroclinic orbits by finding the intersections of the unstable manifold and the stable manifold of the equilibria[9].

Although several methods for obtaining invariants for the system of nonlinear ordinary differential equations(ODEs) like LV system [10-17] are now available in the literature but getting their solutions in closed form (in terms of elementary known function) even for a simpler system is not an easy task. F. GonzalezGascon[11] developed time independent first integrals of LV systems based on the computation of generalized symmetry vectors of the vector field associated with the systems. Aziz[14] discussed local integrability and linearizability of three dimensional LV equations at the origin using the method of Darboux with extensions for inverse Jacobi multipliers and the linearizability of a node in two variables with powerseries arguments in the third variable. Some invariants ware introduced by Almeida et. al.[17] for three species LV system with the aid of Lie Symmetry analysis of the system of ODEs.

In this paper, we consider the LV system of the form[17]

$$
\begin{aligned}
& \frac{d x(t)}{d t}=x(t)\left(d_{1}+c y(t)+z(t)\right) \\
& \frac{d y(t)}{d t}=y(t)\left(d_{2}+x(t)+a z(t)\right) \\
& \frac{d z(t)}{d t}=z(t)\left(d_{3}+b x(t)+y(t)\right) .
\end{aligned}
$$

A substantial number of literatures is dedicated to the integrability properties of these types of LV system[18,19]. It is known that when one of the coupling parameters $a, b, c$ equals unity and growth or decay rate are equal $\left(d_{1}=d_{2}=d_{3}\right)$, the system (1) is completely integrable in the sense that a pair of first integrals exists[18]. Some general solution of this system of ODEs was published by Maier[20] but not as an explicit dependence on $t$. Consequently, getting the analytic expression for heteroclinic orbit between two fixed points is not inevitable.

Purpose of this brief report is to obtain an exact analytic solution of the system of equations (1) to deriving a condition for the existence and time-dependent solution in a compact form of heteroclinic orbits as well as to find the domain in phase-space where heteroclinic orbit exists. We have used separation of variable and the invariant of the system to get an explicit analytic solution containing some parameters involved in the system and presented in section 2. The condition of existence of heteroclinic orbits joining two unstable/saddle or stable/saddle equilibrium points has been proposed in section 3 . Some applications of our result have been exercised in section 4. Our findings have been concluded in section 5. 


\section{Analytic Solution of LV System}

The system of ODEs (1) has five equilibrium points whose dependence on parameters involve in the equations are given by $(i)(0,0,0),(i i)\left(-d_{2},-\frac{d_{1}}{c}, 0\right),(i i i)\left(-\frac{d_{3}}{b}, 0,-d_{1}\right),(i v)\left(0,-d_{3},-\frac{d_{2}}{a}\right)$ and

(v) $\left(-\frac{-a d_{1}+d_{2}+a c d_{3}}{1+a b c},-\frac{a b d_{1}-b d_{2}+d_{3}}{1+a b c},-\frac{d_{1}+b c d_{2}-c d_{3}}{1+a b c}\right)$.

The system of Eqs. (1) has invariant [17]

$$
I=-\frac{1}{a}(y(t)-a z(t)+a x(t)) e^{-\lambda t}
$$

whenever

$$
d_{1}=d_{2}=d_{3}(=\lambda), b=1, c=-\frac{1}{a}
$$

In this case the invariant is nonautonomous, in general, and the number of equilibrium points within finite domain of state space reduces to four given by $(i)(0,0,0)$, (ii) $(-\lambda, a \lambda, 0),(i i i)(-\lambda, 0,-\lambda),(i v)\left(0,-\lambda,-\frac{\lambda}{a}\right)$. The inner equilibrium point of the original system becomes unbounded. The equilibrium point $(0,0,0)$ is unstable one for $\lambda>0$, and stable for $\lambda<0$. The other three equilibrium points are the saddle. It is observed that under the parameter restriction mentioned above all four equilibrium points are on the surface $I=0$.

We introduce here the transformation

$$
x_{1}=x e^{-\lambda t}, y_{1}=y e^{-\lambda t}, z_{1}=z e^{-\lambda t}, \tau=e^{\lambda t} .
$$

Using transformation (4) and the parameter restriction is given in (3), the three species LV system (1) and invariant have the form

$$
\begin{gathered}
\left.\frac{d x_{1}(\tau)}{d \tau}=\frac{x_{1}(\tau)}{\lambda}\left(-\frac{1}{a} y_{1}(\tau)+z_{1}(\tau)\right)\right) \\
\frac{d y_{1}(\tau)}{d \tau}=\frac{y_{1}(\tau)}{\lambda}\left(x_{1}(\tau)+a z_{1}(\tau)\right) \\
\frac{d z_{1}(\tau)}{d \tau}=\frac{z_{1}(\tau)}{\lambda}\left(x_{1}(\tau)+y_{1}(\tau)\right) \\
I=-\frac{\left(y_{1}(\tau)-a z_{1}(\tau)+a x_{1}(\tau)\right)}{a}
\end{gathered}
$$

It is apparent from (8) that the invariant now becomes $\tau$-independent, i.e. autonomous. For the solution of Eqs. (5)-(7) with invariant (8) we consider the following two cases.

(a) Autonomous Invariant $(I=0)$

In this case, the invariant becomes 


$$
z_{1}(\tau)=\frac{y_{1}(\tau)}{a}+x_{1}(\tau)
$$

which represents a plane passing through the origin. It simplifies Eq.(5) into the form of

$$
\frac{d x_{1}(\tau)}{d \tau}=\frac{x_{1}^{2}(\tau)}{\lambda} .
$$

whose solution is given by

$$
x_{1}(\tau)=\frac{-\lambda}{\tau+\lambda k_{1}} .
$$

Here $k_{1}$ is the integration constant. Use of (9) and (11) into Eq.(6) leads to

$$
\frac{1}{y_{1}^{2}(\tau)} \frac{d y_{1}(\tau)}{d \tau_{1}}=\frac{1}{\lambda}-\frac{1+a}{y_{1}(\tau)\left(\tau+\lambda k_{1}\right)} .
$$

so that

$$
y_{1}(\tau)=\frac{\lambda a}{\left(\tau+\lambda k_{1}\right)+\lambda a k_{2}\left(\tau+\lambda k_{1}\right)^{a+1}} .
$$

The symbol $k_{2}$ appearing here is the second integration constant.

Use of (11) and (13) in (9) gives $\tau$ dependence of $z(\tau)$ as

$$
z_{1}(\tau)=\frac{\lambda}{\tau+\lambda k_{1}}\left(\frac{1}{\left(1+\lambda a k_{2}\left(\tau+\lambda k_{1}\right)^{a}\right.}-1\right)
$$

Use of the inverse transformation of (4) in (11), (13) and (14) provides the explicit time-dependent solution of the system of ODEs (1) as

$$
\begin{gathered}
x(t)=\frac{-\lambda e^{\lambda t}}{e^{\lambda t}+\lambda k_{1}} \\
y(t)=\frac{a \lambda e^{\lambda t}}{\left(e^{\lambda t}+\lambda k_{1}\right)+a \lambda k_{2}\left(e^{\lambda t}+\lambda k_{1}\right)^{a+1}} \\
z(t)=\frac{\lambda e^{\lambda t}}{e^{\lambda t}+\lambda k_{1}}\left(\frac{1}{\left(1+\lambda a k_{2}\left(e^{\lambda t}+\lambda k_{1}\right)^{a}\right.}-1\right) .
\end{gathered}
$$

The dependence of integration constant $k_{1}, k_{2}$, and parameters involved in the equations with the initial condition are given by

$$
k_{1}=-\frac{x(0)+\lambda}{\lambda x(0)}
$$




$$
k_{2}=\frac{\{a x(0)+y(0)\} x(0)^{a}}{a(-\lambda)^{1+a} y(0)}
$$

\section{(b) Nonautonomous Invariant $(I \neq 0)$}

In this case, the invariant condition geometrically represents a plane not passing through the origin. Following the steps of previous case $(I=0)$, the explicit time-dependent solution can be found as

$$
\begin{gathered}
x(t)=I x(0) \frac{e^{\lambda t+\frac{I}{\lambda} e^{\lambda t}}}{e^{\frac{I}{\lambda}\{I+x(0)\}-e \frac{I}{\lambda} e^{\lambda t} x(0)}} \\
y(t)=-\frac{a I y(0) c_{1}\left(-\frac{I e^{I / \lambda}}{x(0)}\right)^{a} e^{\frac{I}{\lambda} e^{\lambda t+a} \frac{I}{\lambda} e^{\lambda t}}}{\left(e^{I / \lambda} c_{1}-x(0) e^{\frac{I}{\lambda}} e^{\lambda t}\right)\left\{y(0) e^{\frac{a I}{\lambda} e^{\lambda t}\left(-\frac{I e e^{I / \lambda}}{x(0)}\right)^{a}-e^{\frac{a I}{\lambda}}\left(a c_{1}+y(0)\right)\left(e^{\frac{I}{\lambda} e^{\lambda t}}-\frac{e^{I / \lambda} c_{1}}{x(0)}\right)^{a}}\right\}} \\
z(t)=e^{\lambda t}\left\{\frac{y(t)}{a}+x(t)+I\right\}
\end{gathered}
$$

where $c_{1}=I+x(0)$. From (20)-(22) it appears that each of $x(t), y(t), z(t) \rightarrow+\infty$ or $-\infty$ as $t \rightarrow \infty$ for $\lambda>0$ and $t \rightarrow-\infty$ in case of $\lambda<0$.

\section{Heteroclinic Orbits}

In order to propose the criterion for existence of heteroclinic orbit from the explicit time-dependent solution of system of Eqs.(1) with the restriction on parameter given in (3), we mention here the definition of heteroclinic orbit as: A heteroclinic orbit $\delta(t)$ connects two distinct equilibrium points $E^{i}+$ and $E^{f}$ provided $\lim _{t \rightarrow-\infty} \delta(t)=E^{i}$ and $\lim _{t \rightarrow+\infty} \delta(t)=E^{f}$ or vice versa. Fron $(20)-(22)$ is straightforward to conclude that heteroclinic orbit does not exist for $I \neq 0$.

Consequently, we concentrate on the case of autonomous invariant $I=0$.

\section{Theorem 1}

The solutions of the $L V$ system (1) with parameter restriction (3) and $a \in \mathbb{Z}$ diverges to infinity for $T=$ $\frac{1}{\lambda} \ln \left[\frac{x(0)+\lambda}{x(0)}\right]$ or $T=\frac{1}{\lambda} \ln \left[1+\frac{\lambda}{x(0)}\left\{1-\left(\frac{y(0)}{\operatorname{ax}(0)+y(0)}\right)^{\frac{1}{a}}\right\}\right]$.

Proof. From (15), we observe that the solution diverges to infinity when the denominator of the solutions is zero. If the denominator vanishes at $t=T_{1}$, then $T$ satisfies the equation

$$
e^{\lambda T_{1}}-\frac{\lambda+\mathrm{x}(0)}{\mathrm{x}(0)}=0
$$

leads to 


$$
T_{1}=\frac{1}{\lambda} \log \left[\frac{x(0)+\lambda}{x(0)}\right]
$$

Using the similar condition on $y(t)$ given in (16) one gets equation as

$$
\frac{\left(-\frac{\lambda}{\mathrm{x}(0)}\right)^{-a}(a \mathrm{x}(0)+\mathrm{y}(0))\left(e^{\left.\lambda T-\frac{\lambda+\mathrm{x}(0)}{\mathrm{x}(0)}\right)^{a+1}}\right.}{\mathrm{y}(0)}+e^{\lambda T}-\frac{\lambda+\mathrm{x}(0)}{\mathrm{x}(0)}=0
$$

It is interesting to observe that the equation has two roots. One is $T_{1}$ in (1) and the other is $T_{2}$ given by

$$
T_{2}=\frac{1}{\lambda} \ln \left[1+\frac{\lambda}{x(0)}\left\{1-\left(\frac{y(0)}{a x(0)+y(0)}\right)^{\frac{1}{a}}\right\}\right] \text {. }
$$

Since $z(t)=\frac{y(t)}{a}+x(t)$, it diverges with divergence of either of $x(t)$ or $y(t)$. Hence the theorem is proved.

As mention earlier all four equilibrium points appearing under condition (3) lies on the invariant plane $I=0$. While the equilibrium point $(0,0,0)$ is unstable or stable depending on the sign of $\lambda$, the rest three are saddle in nature. So there may be a heteroclinic connection between $(0,0,0)$ and other saddle equilibrium points. To find the analytic expressions for heteroclinic orbits of the system of Eq.(1) with condition (3) on parameters involve as $t \rightarrow \pm \infty$, the asymptotic behaviour of the solution has been calculated and summarized in Tab-1. While results in the table indicate that the solution approaches two distinct equilibrium points in their two asymptotic limits $t \rightarrow \pm \infty$, Theorem- 1 exhibits the appearance of singularity at some finite time $T_{1}, T_{2}$ in the evolution of $x(t), y(t), z(t)$, thus prohibits the connection of two limiting equilibrium points. If the singularity $\left(T_{1}, T_{2}\right)$ can be removed, then the evolution of the system may terminate at one equilibrium points beginning from close vicinity of some other unstable or saddle equilibrium points.

\begin{tabular}{|c|c|c|}
\hline$\lambda$ and $a$ & $E^{i}$ & $E^{f}$ \\
\hline$a, \lambda>0$ & $(0,0,0)$ & $(-\lambda, 0,-\lambda)$ \\
\hline$a>0, \lambda<0$ & $(-\lambda, 0,-\lambda)$ & $(0,0,0)$ \\
\hline$a<0, \lambda>0$ & $(0,0,0)$ & $(-\lambda, a \lambda, 0)$ \\
\hline$a, \lambda<0$ & $(-\lambda, a \lambda, 0)$ & $(0,0,0)$ \\
\hline
\end{tabular}

Table1: Asymptotic behaviour of LV system with parameter restriction (3) and $a \in \mathbb{Z}$.

Corresponding trajectory in the state space may be regarded as the heteroclinic orbit of the system. Disappearance of singularities can be attributed by claiming that the singularity $T_{1}, T_{2}$ given by Theorem- 1 as a function of parameters in the Eqs.(1)and the initial condition $(x(0), y(0), z(0))$ are complex. Such claim provides conditions for the existence of heteroclinic orbit as

$$
\begin{aligned}
& \text { (i) } \frac{x(0)+\lambda}{x(0)}<0 \text { and } 1+\frac{\lambda}{x(0)}\left\{1-\left(\frac{y(0)}{a x(0)+y(0)}\right)^{\frac{1}{a}}\right\}<0 \text { when } \frac{y(0)}{a x(0)+y(0)}>0 \text { and } \\
& \text { (ii) } \frac{x(0)+\lambda}{x(0)}<0 \text { and } \frac{y(0)}{a x(0)+y(0)}<0 .
\end{aligned}
$$




\section{Applications}

To verify the criterion (i) and (ii) derived in section-3 as a consequence of the principle for the existence of heteroclinic orbit proposed here, we consider four cases where a $(a \in Z)$ and $\lambda$ are positive and negative separately. If the criterion (i) and (ii) involving parameters and initial condition are satisfied, the trajectory connects will two equilibrium points as indicated in Table-1. In Fig.1(i), the marked blue region exhibits domain of existence of heteroclinic orbit predicted by criterion (i) and (ii) for $a=2$ and $\lambda=1$. The green lines show heteroclinic orbits connecting equilibrium points $(-1,0,-1)$ and $(0,0,0)$. The trajectories marked by red lines in Fig.1(i) indicate that whenever the initial point $(x(0), y(0), z(0))$ lies outside the (blue marked) domain of heteroclinic orbit, the trajectory diverges to $( \pm$ ) infinity as $t \rightarrow \infty$. Fig.1(ii), Fig.2(i) and Fig.2(ii) show similar features for parameter value $(a=2, \lambda=-1),(a=-2, \lambda=1)$ and $(a=-2, \lambda=-1)$.

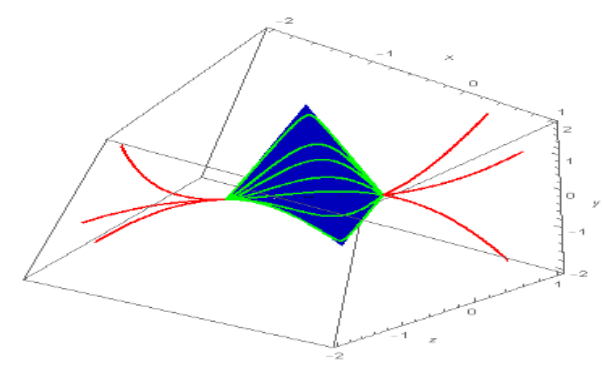

(i)

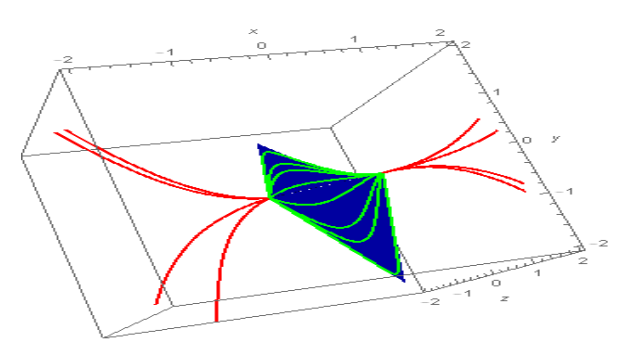

(ii)

Fig.1 Heteroclinic orbit(green line) and domain of existence of heteroclinic orbit (blue region) when (i) $a=2, \lambda=1$ and (ii) $a=2, \lambda=-1$. The red line shows that solution diverges outside the region of heteroclinic orbit for both (i) $a=2, \lambda=1$ and (ii) $a=2, \lambda=-1$.

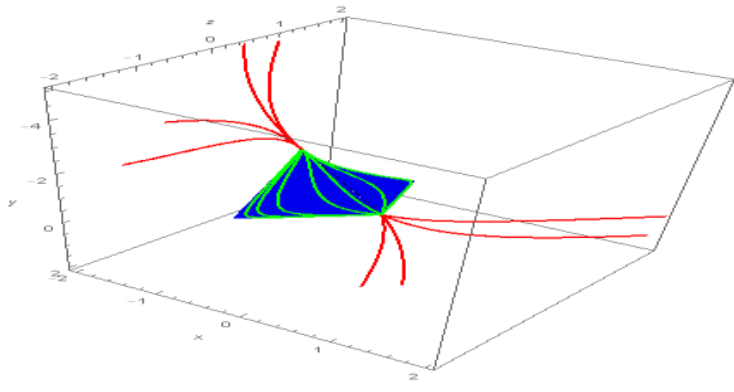

(i)

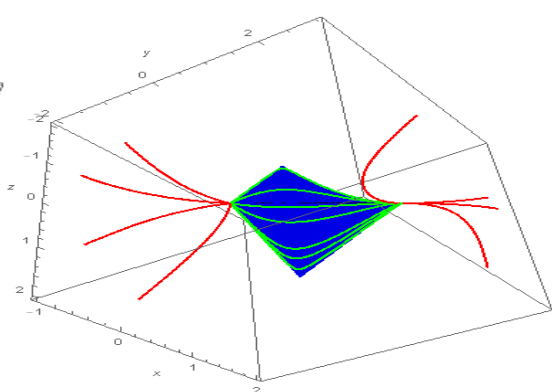

(ii)

Fig. 2 (i) $a=-2, \lambda=1$ and (ii) $a=-2, \lambda=-1$. Descriptions of figures are same as in Fig.1. 
Next, we concentrate on $\lambda<0$, i.e. the growth rate of all three species is negative and $a<0$, i.e. the destructive effect on second species due to interaction with third species. All other inter species interactions are positive. From condition for the existence of heteroclinic orbit we can find heteroclinic orbit of LV system(1) with parameter restriction (3) and $\mathrm{a} \in \mathrm{Z}$ between $(-\lambda, \mathrm{a} \lambda, 0)($ as $\mathrm{t} \rightarrow-\infty)$ and $(0,0,0)($ as $\mathrm{t} \rightarrow+\infty)$.

The above model for $\lambda<0$ and $a<0$ can be applied in a real situation. Here $y$ is considered as human. In the human gut a mutualistic bacteria $E$. coli is present which is considered as $x$. E. coli in human gut takes food for their survival but does not have any bad effect on human rather synthesizes biotin which is essential for humans, there is no outside source of biotin from any food. So perfect mutualistic relationship between $E$. coli and human exists.

Human guts also contain E. histolytica (serious pathogen of human beings) which is considered as z. Human is seriously affected by E. histolytica and suffered from amebiasis.

The relationship of E. coli and E. histolytica are also mutualistic. Enteric bacteria such as E. coli may alter the E. histolytica -induced expression of genes responsible for secretion of $1 \mathrm{~L}-8$. In vitro virulence of $E$. histolytica trophozoites can be increased by $E$. coli. Also, the destruction of cell mono-layer in tissue culture by $E$. histolytica trophozoites has been shown to be increased by bacteria $E$. coli, and in the presence of $E$. histolytica, the growth of $E$. coli is also increased[21].

\section{Conclusions}

We have derived here explicit time and parameter dependent solution in a compact form of LV system with some parameters which may be used as a mathematical model for human pathogen system. Information available from the exact solutions helped us to propose a principle for the existence of heteroclinic orbit for this system. The principle proposed here have been invoked to derive condition relating initial points and parameters involved in this system for the existence of heteroclinic orbit. Such conditions have been used to identify the region in the state space where the heteroclinic orbits connecting equilibrium points of this system may exist. From our exercise, it appears that principle for getting heteroclinic orbit proposed here can be efficiently used for another system too.

Acknowledgements. This work is supported by DST funded research project SR/S2/MS:821/13 dated 24.4.13 and UGC assisted SAP(DRS-Phase-III) program through the Dept. of Mathematics, Visva-Bharati, Santiniketan731235, WB, India.

\section{References}

[1] F. Brauer, C. Castillo-Chavez, Mathematical Models in Population Biology and Epidemiology, Springer, Verlag, 2000.

[2] A. K. Misra, A simple mathematical model for the spread of the spread of two political parties, Nonlinear Analysis: Modelling and Control, 17 (2012) 343-354.

[3] J. Guckenheimer, P. Holmes, Nonlinear Oscillations, Dynamical Systems and Bifurcations of Vector Field, Springer, New York, 1983.

[4] S. Wiggins, Introduction to Applied Nonlinear Dynamical System and Chaos, New York, 1983.

[5] T. Li, G. Chen, G. Chen, On homoclinic and heteroclinic orbit of Chen system, Int. J. Bif. Chaos, 16 (2006) 3035-3041.https://doi.org/10.1142/S021812740601663X

[6] G. Tigan, On a method of finding homoclinic and heteroclinic orbits in multidimensional dynamical systems, Appl. Math. Inf. Sci., 4 (2010) 383-394. 
[7] X. Li, Y. Zhao, C. Sun, The heteroclinic orbits and tracking attractor in a cosmological model with a double exponential potential, Class. Quant. Grav., 22 (2005) 3759-3766.https://doi.org/10.1088/0264$9381 / 22 / 17 / 024$

[8] F. Beiye, The heteroclinic cycles in the model of competition between $\mathrm{n}$-species and its stability, Acta Math. Appl. Sinica, 14 (1998) 404-413. https://doi.org/10.1007/BF02683825

[9] J. Bao, Q. Yang, A new method to find homoclinic and heteroclinic orbits, Appl. Math. Comput., 217 (2011) 6526-6540.https://doi.org/10.1016/j.amc.2011.01.032

[10] P. Gao, Direct integration method and first integrals for three-dimensional Lotka-Volterra systems, Phys. Lett. A, 255 (1999) 253-258.https://doi.org/10.1016/S0375-9601(99)00193-0

[11] F. Gonzalez-Gascon, D. P. Salas, On the first integrals of Lotka-Volterra systems, Phys. Lett. A, 266, (2000), 336-340.https://doi.org/10.1016/S0375-9601(00)00011-6

[12] A. Ballesteros, A. Blasco, F. Musso, Integrable deformations of Lotka Volterra systems, Phys. Lett. A, 375 (2011) 3370-3374.https://doi.org/10.1016/j.physleta.2011.07.055

[13] J. Llibre, C.N. Valls, Polynomial, rational and analytic first integrals for a family of 3-dimensional LotkaVolterra systems, Z. Angew. Math. Phys., 62 (2011) 761-777.https://doi.org/10.1007/s00033-011-0119-2

[14] W. Aziz, C. Christopher, Local integrability and linearizability of three-dimensional Lotka Volterra systems, Appl. Math. Comput., 219 (2012) 4067-4081.https://doi.org/10.1016/j.amc.2012.10.051

[15] N. A. Kudryashov, A.S. Zakharchenko, Analytical properties and exact solutions of the Lotka-Volterra competition system, Appl. Math. Comput., 254 (2015) 219-228.https://doi.org/10.1016/j.amc.2014.12.113

[16] V. S. Varma, Exact solutions for a special prey-predator or competing species system, Bull. Math. Bio. 39 (1977) 619-622.https://doi.org/10.1016/S0092-8240(77)80064-5

[17] M.A. Almeida, M.E. Magalhaes, I.C. Moreira, Lie symmetries and invariants of Lotka-Volterra system, J. Math. Phys., 36 (1999) 1854-1867. https://doi.org/10.1063/1.531362

[18] B. Grammaticos, J. Moulin-Ollagnier, A. Ramani, J-M. Strelcyn, S. Wojciechowski, Integrals of quadratic ordinary differential equations in R3: the Lotka-Volterra system, Physica A, 163 (1990) 683722.https://doi.org/10.1016/0378-4371(90)90152-I

[19] L. Cairo, J. Llibre, Darboux integrability for 3D Lotka-Volterra systems, J. Phys. A: Math. Gen., 33 (2000) 2395-2406.https://doi.org/10.1088/0305-4470/33/12/307

[20] R. S. Maier, The integration of three-dimensional Lotka-Volterra systems, Proc. R. Soc. A, 469 (2013) 0693.http://dx.doi.org/10.1098/rspa.2012.0693

[21] J. M. Kim, H. C. Jung, K. Im, I. S. Song, C. Y. Kim, Synergy between Entamoeba histolytica and Escherichia coli in the induction of cytokine gene expression in human colon epithelial cells. Paras. Res, 84 (1998) 509512.https://doi.org/10.1007/BF03356595 